\title{
Tough Supramolecular Polymer Networks with Extreme Stretchability and Fast Room-temperature Self-healing
}

\author{
Ji Liu ${ }^{1}$, Cindy Soo Yun Tan ${ }^{1,2}$, Ziyi Yu ${ }^{3}$, Nan Li ${ }^{4}$, Chris Abell ${ }^{3}$, Oren A. Scherman ${ }^{1} *^{*}$
}

Synthetic networks are attractive candidates for a wide range of applications, including tissue engineering, drug and protein delivery, sensors, actuators and energy storage. ${ }^{[1-3]}$ Nevertheless, most synthetic networks are soft and brittle, therefore, their practical use in these fields, such as biomaterials, is persistently challenged. Recent reports of several mechanically strong and tough polymer networks have highlighted their potential as structural biomaterials, e.g. cartilage replacement. The toughness typically arises from the combination of molecular engineering and introduction of sacrificial bonds, which can effectively improve the strength as well as material damping and fatigue resistance. ${ }^{[4-9]}$ With the presence of dynamic interactions as sacrificial bonds, the networks are readily processed, bestowing the materials with intriguing self-healing capability and adaptiveness. ${ }^{[3]}$ While several recent reports have illustrated substantial improvements on network toughness and stretchability through molecular complexation with finely tuned association characteristics, ${ }^{[10-12]}$ construction of networks with a rigorous set of those characteristics, like high toughness and extreme stretchability, high mechanical strength and facile roomtemperature self-healing, still remains a big challenge.

We have recently reported a variety of supramolecular polymer networks, consisting of guest-pendant copolymers and $\mathrm{CB}[8]$ host molecules, which exploited the dynamic crosslinking of guest moieties through $\mathrm{CB}$ [8]-mediated hostguest ternary complexations. ${ }^{[13,14]}$ The dynamic nature of $\mathrm{CB}[8]$ host-guest interactions imparts the assembled networks with remarkable responsiveness, arising from the addition of competitive guests, redox change, UV irra-

** Acknowledgment: J.L. is financially supported by the Marie Curie FP7 SASSYPOL ITN (607602) program. C.S.Y.T. thanks the Ministry of Education of Malaysia and MARA University of Technology for their financial supports. Z.Y. and C.A. are funded by the EPSRC, Institutional Sponsorship 2012-University of Cambridge EP/K503496/1 and the Translational Grant EP/H046593/1. O.A.S thanks the EPRSC (EP/F0355351 and EP/G060649/1) and the ERC (ASPiRe, 240629) for their funding.

* 1 Melville Laboratory for Polymer Synthesis, Department of Chemistry, University of Cambridge, Cambridge, CB2 1EW, United Kingdom. E-mail: oas23@cam.ac.uk.

${ }^{2}$ Faculty of Applied Sciences, Universiti Teknologi MARA, 94300 Kota Samarahan, Sarawak, Malaysia.

${ }^{3}$ Department of Chemistry, University of Cambridge, Lensfield Road, Cambridge CB2 1EW, UK.

${ }^{4}$ Department of Chemical Engineering and Biotechnology, University of Cambridge, Tennis Court Road, Cambridge CB2 1QT, UK.

Supporting information for this article is available on the WWW under or from the author. diation and temperature variation, leading to a range of routes to tune the assembly/disassembly. ${ }^{[15,16]}$ However, these supramolecular networks are relatively weak regarding mechanical characteristics. In order to improve them, a higher degree of dynamic crosslinking and/or mass fraction is often desired. Unfortunately, this has been impeded by the limited solubility of $\mathrm{CB}[8]$ and high viscosity of the polymer solutions, resulting in an inhomogeneous system and difficulty in scale-up and manipulation. ${ }^{[17]}$

Here, we explore the in situ polymerisation of a monomer precursor solution, consisting of functional co-monomers, which are complexed with the $\mathrm{CB}[8]$, and acrylamide, to generate $\mathrm{CB}[8]$-based supramolecular polymer networks (Figure 1a), similar to the report of Harada and coworkers by exploiting the copolymerization of $\beta$-cyclodextrin$(\beta-\mathrm{CD})$ and adamantane-based (Ad) monomers. ${ }^{[18]}$ A high $\mathrm{CB}[8]$ host concentration, as well as overall monomer concentration can be readily accessed within the precursor solution, giving access to a higher degree of non-covalent crosslinking and mass fraction of the resultant network, without interference from chain entanglement, high viscosity or limited solubility of the guest-pendant copolymers. Moreover, a great variety of monomers and guest molecules can be adopted to construct the networks, leading to a library of materials with a range of properties. Zhang and coworkers recently reported the fabrication of microgels from a monomer mixture containing $\mathrm{N}$-isopropylacrylamide (NIPAm) and a functional acrylate monomer possessing PheGly-Gly moieties with $\mathrm{CB}[8]$ as supramolecular crosslinkers. ${ }^{[19]}$ In their case, the relative low overall monomer concentration ( $c a .20 \mathrm{mM}$ ) and thermo-induced micellisation of the polyNIPAm chains at $70{ }^{\circ} \mathrm{C}$ (above LCST) resulted in a microgel system as opposed to a macroscopic hydrogel. Here, a substantially higher monomer concentration (up to 2 $\mathrm{M})$ readily achieves a macroscopic network. The dynamic $\mathrm{CB}[8]$ host-guest interactions (Figure 1b) contributes to the networks' toughness through crack bridging by polymer chain entanglement and sliding, as well as energy dissipation by reversible and simultaneous association/dissociation of the host-guest complexes. Therefore, it could impart the networks with extreme stretchability and astounding toughness.

To probe the minimum monomer concentration $\left(C_{m o n}\right.$, $C_{\text {mon }}=C_{\text {guest }}+C_{\text {acrylamide }}$ ) required for network formation, a series of polymerisations were carried out, with overall $C_{m o n}$ ranging from 0.05 to $2.0 \mathrm{M}$, while keeping the same fraction of $\mathrm{CB}[8]$ host-guest complexes $\left(C_{\text {guest }} / C_{\text {acrylamide }}=\right.$ 
a

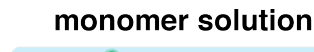

b
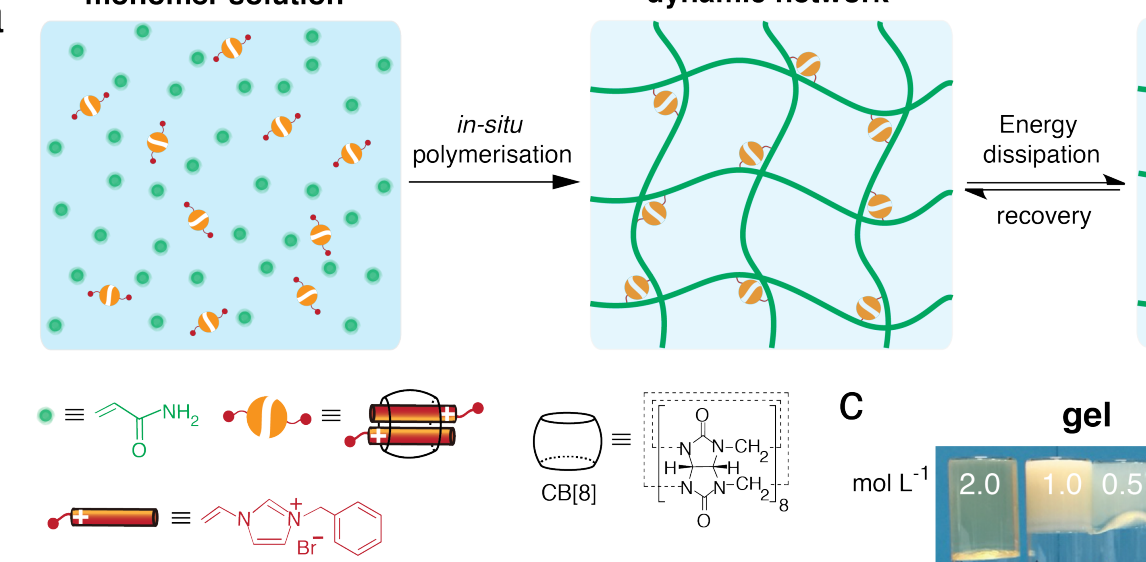

C

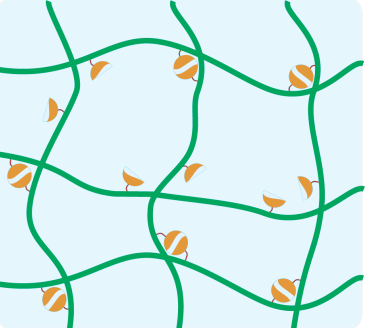

gel

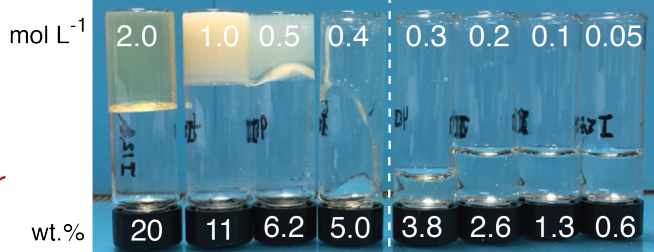

Figure 1. a) In situ polymerisation of supramolecular polymer networks in the presence of macrocyclic host cucurbit[8]uril (CB[8]) and polymerisable guest molecules (1-benzyl-3-vinylimidazolium) and acrylamide. The CB[8] supramolecular ternary conjugates serve as dynamic crosslinks, leading to a network structure after photo-initiated polymerisation at room temperature. On the other hand, they also act as sacrificial bonds that rupture under deformation and dissipate energy, which can further re-form, resulting in the self-healing of macroscopic supramolecular network. b) Stepwise formation of ternary host-guest complexation between $\mathrm{CB}[8]$ and guest molecules, following an 1:2 binding mode. c) Photographs of the supramolecular polymer networks with different monomer concentration $\left(\mathrm{mol} \mathrm{L}^{-1}\right)$ or mass fraction $(w t . \%)$, as well as the corresponding inverted-vial demonstration of the viscosity.

$\left.1 / 19 ; C_{\text {guest }} / C_{C B[8]}=2 / 1\right)$. The network samples were initially examined by an inverted-vial test (Figure 1c). An increase in viscosity was observed for $C_{m o n}>0.3 \mathrm{M}$, while stable networks were formed above $0.5 \mathrm{M}$. This indicates that $\mathrm{CB}[8]$ host-guest interactions among the polymer chains are stabilised at high mass fraction to yield microscopically stable supramolecular networks, even without any chemical crosslinkings. The concentration-dependent gelation is partially attributed to the chain entanglement, corroborated by the estimated overlap concentration of $c^{*}$ of $26.3 \mathrm{~g} \mathrm{~L}^{-1}$ (0.4 M, See ESI Figure $\mathbf{S 4}$ and discussion). Unlike the dual network consisting of both $\mathrm{CB}[8]$ crosslinking and covalent crosslinking, which experiences a high-degree swelling (ca. $100 \times$ by weight), ${ }^{[20]}$ complete dissolution of the $\mathrm{CB}[8]$ network here in Milli-Q water $\left(25^{\circ} \mathrm{C}, 18 \mathrm{~m} \Omega\right)$ after five days stirring evidences that no covalent crosslinking exists. A fluffy and amorphous material was obtained upon freeze-drying, and subsequently led to the reformation of supramolecular polymer networks through dissolution (See ESI Figure S2). The critical gelation concentration can be downshifted by introducing a small amount of covalent crosslinker, such as $N, N^{\prime}$-bismethylenebisacrylamide. [20]

Nominal tensile stress-strain curves of the supramolecular polymer networks (Figure 2a) demonstrate that an increase in $C_{m o n}$ to $2 \mathrm{M}$ leads to strength enhancement; and this network sample is strong enough to readily lift a weight $2000 \times$ greater than its own (see ESI Figure S5e). The $\mathrm{CB}$ [8]-based networks prepared from lower monomer concentrations (e.g. $\left.C_{m o n}=0.5 \mathrm{M}\right)$ do not exhibit an obvious yield point, because of insufficient chain entanglement, whereas networks from higher concentrations (e.g. $C_{\text {mon }}=$
$2 \mathrm{M})$ exhibit clear yield points with a markedly increased modulus. Likely, the enhanced strength is attributed to sufficient chain entanglement, coupled with $\mathrm{CB}$ [8]-based noncovalent crosslinkings. Control polymerisations with $\mathrm{CB}$ [7] or in the absence of $\mathrm{CB}[n]$ only result in viscous fluids, with a lower rheological modulus by at least two orders of magnitude (See ESI Figure S9 and discussion). It is of interest to note that all dumbbell-shaped $\mathrm{CB}[8]$-based network samples can be stretched beyond $45 \times$ their original dimension (maximum stretch that could be obtained from the tensile apparatus) without fracture observed, regardless of the starting $C_{m o n}$.

Recently, Vlassak and coworkers reported synthesis of a network through the micellar copolymerisation of a 2ureido-4-pyrimidone (UPy)-containing monomer with acrylamide, which can be stretched over $100 \times$ its initial length from a ribbon-shaped sample. ${ }^{[1]}$ When our CB[8]-based network sample (of the same dimension) was subjected to similar test (see ESI Figure S7), a fracture strain of $107 \times$ the initial length, with a nominal fracture stress of ca. 1.8 MPa detected, confirming the as-claimed extreme stretchability and high strength of these $\mathrm{CB}[8]$-based networks. As far as known to us, this mechanical strength is comparable to, not to say much higher than, previouslyreported aqueous network with a maximum strain up to $100 .^{[11,21]}$

The deformation-rate dependent tensile behaviour also confirm the chain dynamics as described in Figure 1a. A pronounced increase in both stress and Young's modulus is detected $\left(24 \times\right.$ from $0.016 \mathrm{MPa}$ at $10 \mathrm{~mm} \mathrm{~min}^{-1}$ to $0.42 \mathrm{MPa}$ 

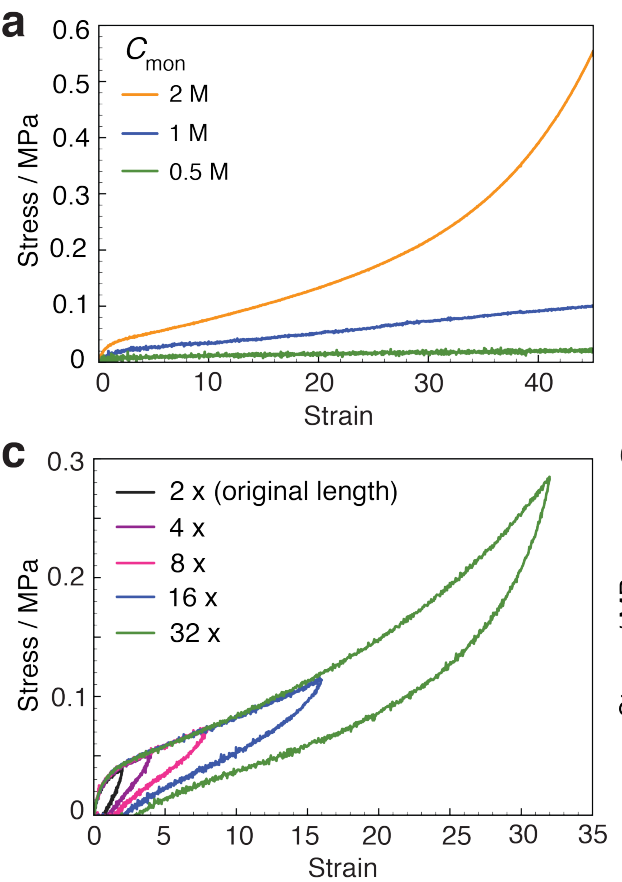
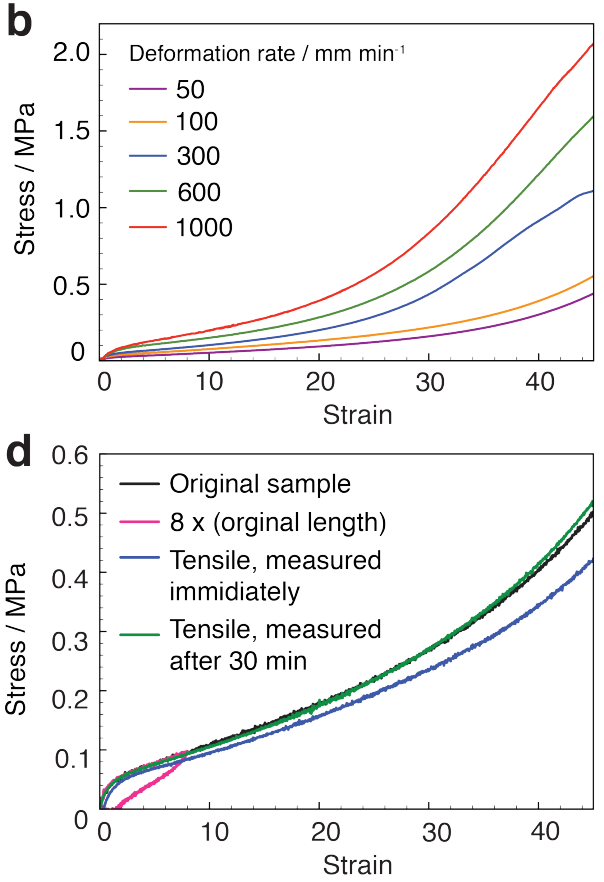

Figure 2. a) Uniaxial tensile behaviour of the supramolecular polymer networks with different monomer concentrations $\left(C_{m o n}\right)$. b) Uniaxial tensile behaviour of the supramolecular polymer networks $\left(C_{\text {mon }}\right.$ of $2 \mathrm{M}$ ) under different deformation rates ranging from 50 to $1000 \mathrm{~mm} \mathrm{~min}^{-1}$ (note: all of the samples reached the maximum strain possible on the tensile machine without fracture). c) Samples of the supramolecular polymer network $\left(C_{m o n}\right.$ of 2 M) were subjected to a cycle of loading and unloading to various strains (deformation rate of $100 \mathrm{~mm} \mathrm{~min}^{-1}$ ). d) Uniaxial tensile test of the supramolecular polymer networks $\left(C_{m o n}\right.$ of $2 \mathrm{M}$ ) immediately (blue curve) after the stretching/retracting cycle (strain of $8 \times$, red curve), and the sample which was left to self-repair for $30 \mathrm{~min}$ at room temperature (green curve). The level of overlap between the normal run (black curve) and the self-repaired sample after 30 min (green curve) indicates complete self-repairing of the network structures via the re-formation of supramolecular host-guest complexation.

at $2000 \mathrm{~mm} \mathrm{~min}^{-1}$ ) (Figure 2b and ESI Figure S6), attributing to the dynamic and reversible features of $\mathrm{CB}[8]$ hostguest interactions. No fracture was observed again up to a strain of $45 \times$ (the maximum stretch that could be achieved with the tensile apparatus), regardless of the deformation rates (from 10 to $2000 \mathrm{~mm} \mathrm{~min}^{-1}$ ). This unique viscoelastic character contributes to the networks' high shock-absorption capability and toughness. ${ }^{[20]}$ A ball-drop test was carried out by dropping a metal ball (16 g) onto the network membrane from a height of $35 \mathrm{~cm}$ (See ESI Supporting Movie 1). When the dropping ball impacted the membrane, a large deformation was observed before complete re-bounding. The membrane recovered to its initial flat configuration after dissipating the energy. While metal ball with even higher impacting energy (drop height of $50 \mathrm{~cm}$ ) could lead to failure of the network membrane (See ESI Supporting Movie 2). Similar shock-absorption characteristics were also observed when dropping a ball made of the CB[8]-based network onto a glass substrate (See ESI Supporting Movie 3). A smaller re-bounding height was detected for the $\mathrm{CB}[8]$ based network ball, compared with its analogue with covalent crosslinking.

To further investigate the viscoelastic properties of these $\mathrm{CB}[8]$ supramolecular polymer networks, we performed cyclic tensile tests. As shown in Figure 2c, clear yield is observed upon loading the sample to a predetermined strain, with a hysteresis and apparent residual strain observed when retracted to zero stress. The residual strain and large hysteresis reveal that some structural changes are preserved after the unloading process, however, they gradually decreased to zero after a certain waiting time. As shown in Figure 2d, a decrease in both modulus and stress was detected in the tensile measurement immediately after the cyclic tensile test at a strain of $8 \times$. However, after a waiting period of $30 \mathrm{~min}$, the sample recovers all of its initial mechanical properties, as evidenced by an overlap of the tensile curves. These results indicates that even without any covalent crosslinking, chain sliding does not occur under the large deformation as the host-guest complexes are widely distributed throughout the network. In this network system, the $\mathrm{CB}[8]-$ mediated host-guest complexes serve as reversible sacrificial bonds, which could break and reform dynamically, giving high toughness to these viscoelastic supramolecular polymer networks. Although several networks of high strength and toughness based on physical association have recently been developed, ${ }^{[9,22,23]}$ they are only capable of partial recovery, likely due to some amount permanent damage of covalent bonds upon loading or a greatly limited self-healing rate due to high modulus of the materials, thus retarded chain mobility. Thus, a delicate balance between the soft and ductile characters arising from the non-covalent interactions and 
a

b
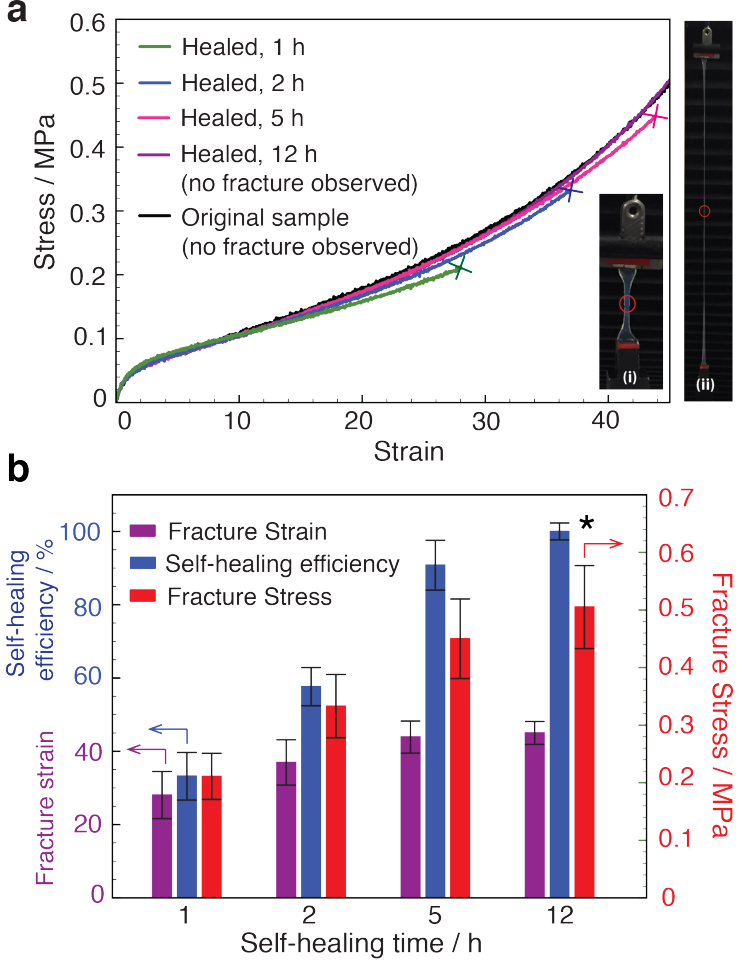
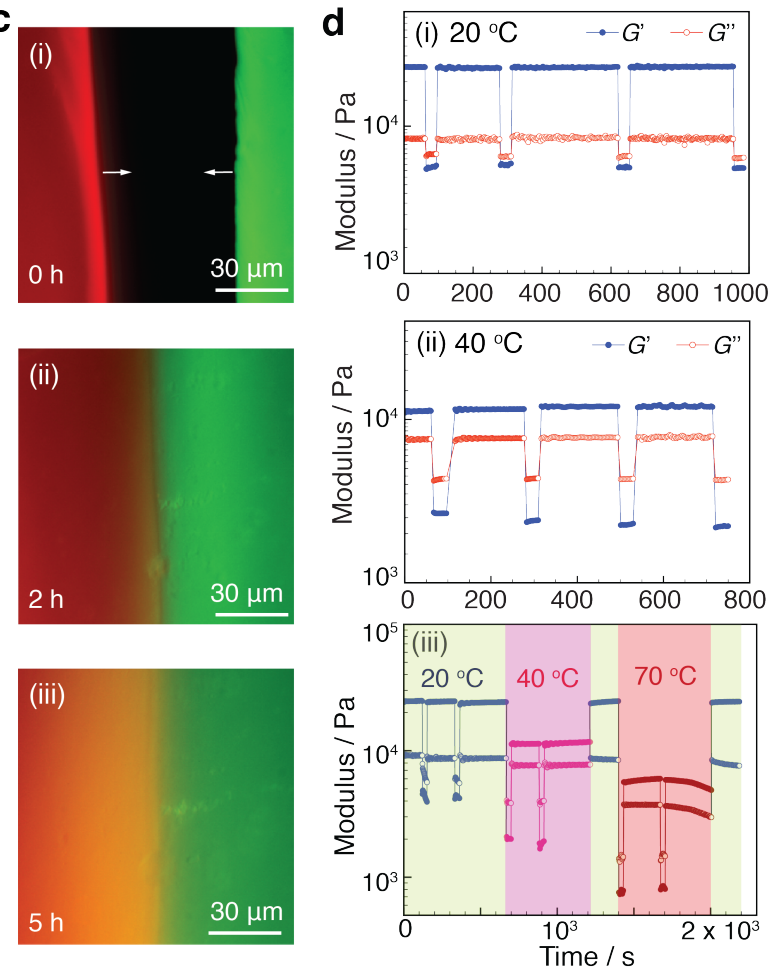

Figure 3. a) Stress-strain curves of the virgin and self-healed supramolecular polymer network $\left(C_{m o n}\right.$ of $\left.2 \mathrm{M}\right)$ after different healing times at $25^{\circ} \mathrm{C}$ (note: the original sample and self-healed sample after $12 \mathrm{~h}$ did not fracture at strain of $45 \times$, which is the maximum strain achieved by the tensile machine); inset: photographs demonstrating the self-healed sample during stretching at a deformation ratio of $\lambda=0$ (i) and $\lambda=28$ (ii). The red circles refer to the cut location of a self-healed sample $(1 \mathrm{~h}$ ) before and after stretching to a strain of $28 \times$. b) Plotting of fracture stress, fracture strain as well as self-healing efficiency against self healing time (* for the 12-h self-healed sample, nominal stress at $45 \times$ was taken as the fracture stress). c) Indirectly demonstration of the self-healing via fluorescence microscopic images of two supramolecular polymer networks, one chemically-labeled with rhodamine (red), the other with fluorescein (green). Two network films $(5 \mathrm{~m}(W) \times 5 \mathrm{~mm}(L) \times 50 \mu \mathrm{m}(T))$ were brought together (contact surface: $5 \mathrm{~mm} \times 50 \mu \mathrm{m})$ with a gap of ca. $50 \mu \mathrm{m}$ (black area, i), self-healing at room temperature for $2 \mathrm{~h}$ (ii) and $5 \mathrm{~h}$ (iii). The appearance of the light yellow area confirms the self-healing at the contact region. d) Continuous step-strain measurements of the network sample at $20{ }^{\circ} \mathrm{C}$ (i) and $40{ }^{\circ} \mathrm{C}$ (ii), and also consecutive step-strain sweeping with the network sample subjected to heating/cooling cycles among 20,40 and $70{ }^{\circ} \mathrm{C}$ (iii) (high-amplitude oscillatory parameters: strain $\dot{\gamma}=500 \%$, angular frequency $\omega=10 \mathrm{rad} \mathrm{s}^{-1}$, low-amplitude oscillatory parameters: $\dot{\gamma}=0.5 \%, \omega=10 \mathrm{rad} \mathrm{s}^{-1}$ ).

rigid and brittle domain from the covalent bonds is crucial for formation of tough hydrogels, which can readily selfrepaired.

Traditional covalent networks are too brittle to sustain compression, slicing or elongation. ${ }^{[3]}$ Exploiting CB [8] host-guest complexation for energy dissipation, the networks are highly elastic and stretchable, behaving like a rubber. The samples can be manipulated with high levels of deformation, including twisting, knotting and extensive stretching (see ESI Figures S5b, S5c and S5d), and could not be readily sliced with a blade (see ESI Figure S5a). The supramolecular materials are extremely notch-insensitive, and the virgin network sheet could be stretched to $52 \times$ before rupture (see ESI Figure S5g and ESI Supporting Movie 4) with relatively high transparency (See ESI Figure S8), while subsequent fracture was observed near the metal clamp, where intensive stress accumulates. In the case of notched samples, the notch area was dramatically blunted and remained stable (See Figure S5h and ESI Supporting Movie 5) up to a stretch ratio of $40 \times$, after which a crack initiated at the front of the notch, and rapidly propagated through the entire sample.

Supramolecular polymer networks, assembled through non-covalent and directional interactions, such as $\mathrm{H}$ bonding, $\pi-\pi$ stacking, metal-ligand complexation and host-guest interactions, are imparted with a wide range of stimuli-responsive features, including mechanochromism, bioactivity and mechanical morphing, on account of their dynamic nature. ${ }^{[3,24]}$ A major challenge in the field of selfhealing polymers is to combine robust mechanical properties and high self-healing efficiency under mild conditions. ${ }^{[24,25]}$ Currently, most spontaneously and intrinsically self-healable materials are mainly focused on relatively weak polymeric systems due to the high reliance on chain dynamics. Moreover, most of the fast self-healing is activated by heating, high-intensity UV irradiation, moisture or addition/release of curing reagents, which might further hindering their potential applications. ${ }^{[24]}$ Here, room-temperature self-healing of the $\mathrm{CB}$ [8]-based supramolecular polymer networks was studied with dumbbell-shaped samples, which were cut into 

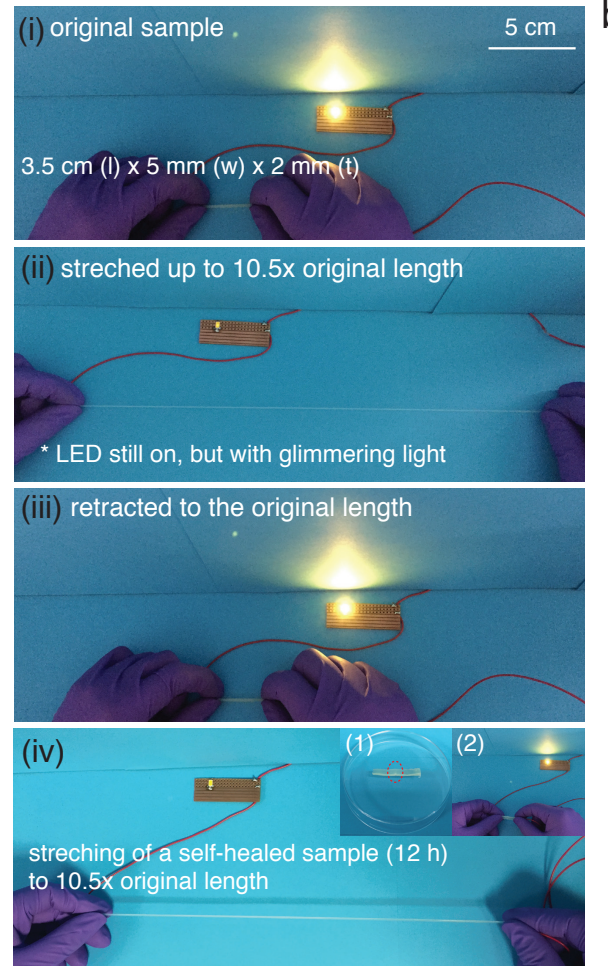

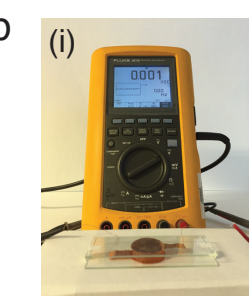

original sample
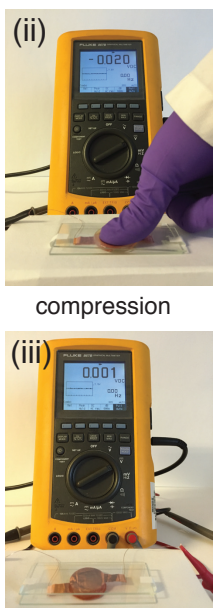

recovery

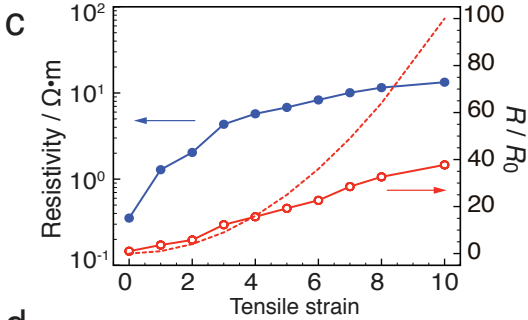

d

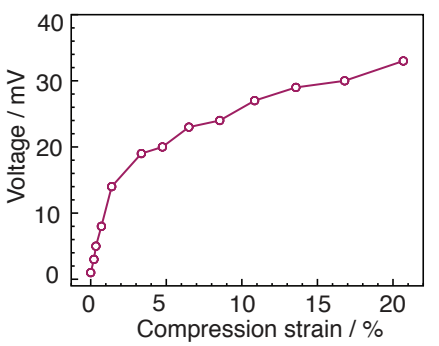

e

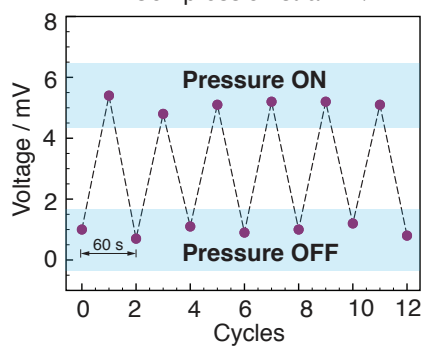

Figure 4. a) Demonstration of the supramolecular polymer network as conductor to light LED bulb when connected with a battery, before (i) and after (ii) the sample was stretched to a strain $\lambda=10.5$, recovered to its original length (iii), as well as using of a self-healing sample (12 h, iv-1) as a conductor, before (iv-2) and after (iv) stretching to a strain of $\lambda=10.5$. b) Demonstration of the pressure sensing with the network sample connected to a multimeter (voltage channel), before (i) and upon (ii) compression ( $\mathrm{ca}$. 30\% deformation), as well as compression removal and voltage signal recovered to the original value within $18 \mathrm{~s}$ (iii). c) Dependence of the resistivity and resistance $\left(R / R_{0}\right)$ of the network sample when stretched to variant strains. d) Plotting of the voltage signal against compression pressure. e) Evolution of the voltage signal of a network sample during a cyclic compression/decompression cycles upon a compression strain of $2 \%$ (stress of $c a$. $0.35 \mathrm{kPa}$ ), with an interval of $60 \mathrm{~s}$ for each cycle.

two pieces and then brought back into contact for variable self-healing periods. The healing efficiency reached $35 \%$ after $1 \mathrm{~h}$ at $25{ }^{\circ} \mathrm{C}$, and the healed joint was able to withstand large stress $(40 \%$ of the original stress, Figure 3a) at a strain of $28 \times$, which is subsequently followed by fracture at the healed region (Figures 3a-ii and Figures 3a-iii, marked with the red circles). A longer self-healing period, i.e. $12 \mathrm{~h}$, can lead to complete self-healing at room temperature. This efficient self-healing at $25^{\circ} \mathrm{C}$, without extra treatment, is comparable to previously reported self-healing systems based on H-bonding, ${ }^{[25-29]}$ metal-ligand conjugation, ${ }^{[30,31]}$ electrostatic ${ }^{[8]}$ and host-guest interactions, ${ }^{[18,32]}$ to cite only a few. Adhesion between two uncut surfaces was also observed through fluorescence microscopy to ascertain the indirect self-healing. As shown in Figures 3c, the appearance of a yellow area, corresponding to the overlap of red (RITC) and green luminescence (FITC), corroborates the indirect self-healing in the contact area. The microscopic, or even molecular-scale, self-healing/repairing capability was also observed in rheological step-strain measurement, where a large-amplitude sweep $\left(\gamma=500 \%, \omega=10 \mathrm{rad} \mathrm{s}^{-1}\right)$ induces the destruction of network structure $\left(G^{\prime}<G^{\prime \prime}\right)$. Prompt recovery of the network was observed during the subsequent small-amplitude sweep $\left(\gamma=0.5 \%, \omega=10 \mathrm{rad} \mathrm{s}^{-1}\right)$ at $20^{\circ} \mathrm{C}$ (Figure 3d-i), $40^{\circ} \mathrm{C}$ (Figure 3d-ii) and even $70^{\circ} \mathrm{C}$ (See ESI Figure S14). Alternating step-strain sweeps at different temperatures (Figure 3d-iii) also evidence that the self-repairing is unaffected by the heating-cooling cycles, in agreement with the high thermal stability of the networks detected as shown in Figure S13.

Aqueous networks are inexpensive and easy to make, ideal for demonstrating conceptual designs and fabricating devices with desirable functions. The introduction of $\mathrm{CB}[8]$-based host-guest complexes here effectively imparts the networks with excellent mechanical performance and good transparency during stretching (see ESI Figure S8); while mobile ions within the materials enable the percolation of conductive pathways. Compared with most electronic conductors, such as metals and carbon-based materials, hydrogel-based ionic conductors exhibit higher resistivity. ${ }^{[33]}$ They become more attractive in the cases which 
require high stretchability, self-healability and transparency, such as wearable electronics, flexible devices and batteries. Figure 4a and ESI Supportive Movie 6 demonstrate that the $\mathrm{CB}[8]$-based networks are ionic conductive, sustaining a LED light, even when stretched to $10.5 \times$ (Figure 4aii). Resistance increased by a factor of two once it is stretched by $10 \times$, due to the elongated length and decreased cross-sectional area (Figure 4c). However, unlike alginatesystems reported by Suo and Whitesides, ${ }^{[33]}$ the resistance ratio here $\left(R / R_{0}\right)$ does not follow the tendency of $\lambda^{2}$ (dashed red line in Figure 4c) when it reaches higher strains $(\lambda>$ $4)$, due to the increase in resistivity upon stretching ( 0.3 to $10 \Omega \cdot \mathrm{m})$. This can be explained by the limited available ions within in the $\mathrm{CB}$ [8]-based network samples $\left(\mathrm{Br}^{-} c a .0 .1 \mathrm{M}\right)$, thus, stretching of the networks could significantly increase the resistivity (blue line in Figure 4c).

Deformation-induced variation in resistance can be readily exploited as a pressure sensor to monitor the applied force. Compressing the network promptly induces a change in voltage, while removal of applied force is accompanied with facile recovery of initial voltage signal (Figure 4b and ESI Supportive Movie 7). Quantitative analysis was conducted with the simple compression apparatus, which was equipped with a multimeter, whereby pressure vs. voltage was recorded (Figure 4d). Cyclic compression/decompression (strain of $2 \%$ ) led to repeatable voltage changes (Figure 4e). Inherent ionic conductivity of the flexible yet tough transparent networks opens the possibility to construct robust interfaces between ionic/biological and ionic/electronic systems.

In summary, we demonstrated here the $\mathrm{CB}[8]$-based supramolecular network through in situ polymerisation in the presence of $\mathrm{CB}[8]$ host molecules with remarkable mechanical performances. Small amount of $\mathrm{CB}[8] /$ guest complexes served as dynamic cross-linkers $(2.5 \mathrm{~mol} . \%)$ to dramatically improve mechanical properties, as well as energy dissipation components to impart integrity with astounding toughness, stretchability and fast room-temperature selfhealing. This kind of supramolecular polymer network, with a synthetically simple approach and outstanding mechanical properties, should dramatically increase the choice of tough hydrogels for numerous applications, such as biomaterials, sensors and wearable electronic devices.

\section{References}

[1] J. R. Capadona, K. Shanmuganathan, D. J. Tyler, S. J. Rowan, C. Weder, Science 2008, 319, 1370-1374.

[2] A. Miserez, T. Schneberk, C. Sun, F. W. Zok, J. H. Waite, Science 2008, 319, 1816-1819.

[3] T Aida, E. Meijer, S. Stupp, Science 2012, 335, 813-817.

[4] H. Lee, S. M. Dellatore, W. M. Miller, P. B. Messersmith, Science 2007, 318, 426-430.

[5] H. Lee, B. P. Lee, P. B. Messersmith, Nature 2007, 448, 338-341
[6] P. Y. Dankers, T. M. Hermans, T. W. Baughman, Y. Kamikawa, R. E. Kieltyka, M. Bastings, H. M. Janssen, N. A. Sommerdijk, A. Larsen, M. J. van Luyn, et al., Adv. Mater. 2012, 24, 2703-2709.

[7] M. Guo, L. M. Pitet, H. M. Wyss, M. Vos, P. Y. Dankers, E. Meijer, J. Am. Chem. Soc. 2014, 136, 6969-6977.

[8] T. L. Sun, T. Kurokawa, S. Kuroda, A. B. Ihsan, T. Akasaki, K. Sato, M. A. Haque, T. Nakajima, J. P. Gong, Nat. Mater. 2013, 12, 932937.

[9] J.-Y. Sun, X. Zhao, W. R. Illeperuma, O. Chaudhuri, K. H. Oh, D. J. Mooney, J. J. Vlassak, Z. Suo, Nature 2012, 489, 133-136.

[10] F. Luo, T. L. Sun, T. Nakajima, T. Kurokawa, Y. Zhao, K. Sato, A. B. Ihsan, X. Li, H. Guo, J. P. Gong, Adv. Mater. 2015, 27, 2722-2727.

[11] I. Jeon, J. Cui, W. R. Illeperuma, J. Aizenberg, J. J. Vlassak, Adv Mater. 2016, 28, 4678-4683.

[12] H. J. Zhang, T. L. Sun, A. K. Zhang, Y. Ikura, T. Nakajima, T. Nonoyama, T. Kurokawa, O. Ito, H. Ishitobi, J. P. Gong, Adv. Mater. 2016, 28, 4884-4890.

[13] E. A. Appel, F. Biedermann, U. Rauwald, S. T. Jones, J. M. Zayed, O. A. Scherman, J. Am. Chem. Soc. 2010, 132, 14251-14260.

[14] C. S. Tan, J. del Barrio, J. Liu, O. A. Scherman, Polym. Chem. 2015, 6, 7652-7657.

[15] J. Liu, C. S. Y. Tan, Y. Lan, O. A. Scherman, Macromol. Chem. Phys. $\mathbf{2 0 1 5}, 217,319332$

[16] J. Liu, Y. Lan, Z. Y. Yu, C. S. Y. Tan, R. M. Parker, C. Abell, O. A. Scherman, Acc. Chem. Res. 2017, DOI:10.1021/acs.accounts.6b00429.

[17] M. J. Rowland, M. Atgie, D. Hoogland, O. A. Scherman, Biomacromolecules 2015, 16, 2436-2443.

[18] M. Nakahata, Y. Takashima, A. Harada, Macromol. Rapid Commun. 2016, 37, 86-92.

[19] Q. Song, Y. Gao, J.-F. Xu, B. Qin, M. J. Serpe, X. Zhang, ACS Macro Lett. 2016, 5, 1084-1088.

[20] J. Liu, C. S. Y. Tan, Z. Y. Yu, Y. Lan, C. Abell, O. A. Scherman, Adv. Mater. 2017, DOI:10.1002/adma.201604951.

[21] G. Sun, Z. Li, R. Liang, L.-T. Weng, L. Zhang, Nat. Commun. 2016 , 7, 12095 .

[22] M. A. Haque, T. Kurokawa, G. Kamita, J. P. Gong, Macromolecules 2011, 44, 8916-8924.

[23] Y. Tanaka, R. Kuwabara, Y.-H. Na, T. Kurokawa, J. P. Gong, Y. Osada, J. Phys. Chem. B 2005, 109, 11559-11562.

[24] Y. Yang, M. W. Urban, Chem. Soc. Rev. 2013, 42, 7446-7467.

[25] P. Cordier, F. Tournilhac, C. Soulié-Ziakovic, L. Leibler, Nature 2008, 451, 977-980.

[26] S. R. White, N. Sottos, P. Geubelle, J. Moore, M. Kessler, S. Sriram, E. Brown, S Viswanathan, Nature 2001, 409, 794-797.

[27] Y. Chen, A. M. Kushner, G. A. Williams, Z. Guan, Nat. Chem. 2012, 4, 467-472.

[28] C. Wang, H. Wu, Z. Chen, M. T. McDowell, Y. Cui, Z. Bao, Nat. Chem. 2013, 5, 1042-1048.

[29] B. C. Tee, C. Wang, R. Allen, Z. Bao, Nat. Nanotechnol. 2012, 7, $825-832$.

[30] C.-H. Li, C. Wang, C. Keplinger, J.-L. Zuo, L. Jin, Y. Sun, P. Zheng, Y. Cao, F. Lissel, C. Linder, et al., Nat. Chem. 2016, 8, 618-624.

[31] M. Burnworth, L. Tang, J. R. Kumpfer, A. J. Duncan, F. L. Beyer, G. L. Fiore, S. J. Rowan, C. Weder, Nature 2011, 472, 334-337.

[32] M. Nakahata, Y. Takashima, H. Yamaguchi, A. Harada, Nat. Commun. 2011, 2, 511 .

[33] C. Keplinger, J.-Y. Sun, C. C. Foo, P. Rothemund, G. M. Whitesides, Z. Suo, Science 2013, 341, 984-987. 